\title{
Sustainable Development in Block Random Systems
}

By Ferenc Juhász

\section{Budapest University of Technology and Economics}

Abstract- In paper [1], stability of a block random model was studied as a possible model for economic systems. Crisis means significant and quick change in the number of participants of a system. It was proved that a smaller system is more stable than a larger one with the same parameters. Further, the number of participants can significantly alter without any outer interactions resulting in crisis.

In paper [2], stability properties of a block random model with fixed number of participants was investigated. It was studied, that how two parameters of the model, density matrix and dispersion influence behavior of the system. It was shown that proportionally smaller in absolute value density matrix results in a shorter cycle time. Also larger dispersion makes the cycle time shorter. It was suggested that a longer cycle time makes it possible the participants to adapt themselves to circumstances and thus to avoid crises. In this case repeated recessions and growths appear which can be called structural cycles.

In the present paper we investigate connection between real parameters of economy and parameters of the block random model. We point out that base rate bounded by an appropriate level is useful for working the system without any crisis. As a result of these studies, it has become clear that sustainable development can be defined in terms of avoiding crisis rather than achieving growth.

Keywords: random matrices, eigenvalues, lyapunov stability, economics, ecology.

\section{GJCST-G Classification: F.1.1}

Strictly as per the compliance and regulations of:

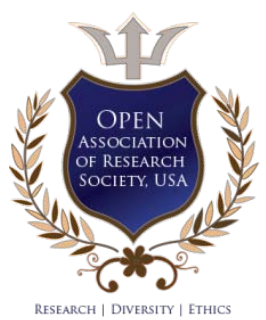

(C) 2020. Ferenc Juhász. This is a research/review paper, distributed under the terms of the Creative Commons AttributionNoncommercial 3.0 Unported License http://creativecommons.org/licenses/by-nc/3.0/), permitting all non-commercial use, distribution, and reproduction in any medium, provided the original work is properly cited. 


\section{Sustainable Development in Block Random Systems}

Ferenc Juhász

Abstract- In paper [1], stability of a block random model was studied as a possible model for economic systems. Crisis means significant and quick change in the number of participants of a system. It was proved that a smaller system is more stable than a larger one with the same parameters. Further, the number of participants can significantly alter without any outer interactions resulting in crisis.

In paper [2], stability properties of a block random model with fixed number of participants was investigated. It was studied, that how two parameters of the model, density matrix and dispersion influence behavior of the system. It was shown that proportionally smaller in absolute value density matrix results in a shorter cycle time. Also larger dispersion makes the cycle time shorter. It was suggested that a longer cycle time makes it possible the participants to adapt themselves to circumstances and thus to avoid crises. In this case repeated recessions and growths appear which can be called structural cycles.

In the present paper we investigate connection between real parameters of economy and parameters of the block random model. We point out that base rate bounded by an appropriate level is useful for working the system without any crisis. As a result of these studies, it has become clear that sustainable development can be defined in terms of avoiding crisis rather than achieving growth.

Keywords: random matrices, eigenvalues, lyapunov stability, economics, ecology.

\section{INTRODUCTION}

$\mathrm{t}$ is widely accepted that behavior of economy is quasi cyclic. One can observe time to time appearing small cycles which can be called structural cycles to which participants can easily adapt themselves. Besides, so called crisis cycles manifest too which can be interpreted as large and quick alteration in the number of participants. Accommodation to these changes is problematic, a certain amount of participants are unable to do that.

If one thinks of important parameters of economy as interests rates, incomes and wages, corporate profits, inflation, etc. it seems unclear why systems cannot work similarly with different but proportional level of these parameters.

It is widely known among economists that interest rates play crucial role in successful or less successful behavior of economic systems. In the present paper we draw attention to the connection between structural cycle time and level of interests.

Author: Department of Computer Science and Information Theory Budapest University of Technology and Economics.

e-mail: fjuhasz@t-online.hu

\section{Block Random Model}

We briefly summarize description of the model presented in [1]. The block the random model handles not only deterministic but random effects too. Its behavior depends on two parameters, density matrix and the dispersion of entries. The first relates to expected values, the second to variances of the entries. The model is piecewise linear which makes it possible to handle it easily while non-linearity is taken into account too.

\section{a) Economic model}

Denote $X$ the $n$-dimensional space which we call Capital space and $Y$ the $n$-dimensional space which is called Profit space. Coordinates of point $x \in X$ and $y \in Y$ are the capitals and profits of the participants, respectively.

Let us suppose that we know the $R^{n} \rightarrow R^{n}$ function $F$ which maps the points of $X$ to the points of $Y$. If we linearize $F$ at time zero we get the following expression

$$
y(t) \approx F(x(0))+L(x(t)-x(0))
$$

Where Jacobian $L$ consists of partial derivatives of function $F$.

Let us differentiate by $t$, then we get $\dot{y}(t)=L \dot{x}(t)$ If we take into consideration that profit is change of capital, we get our main equation:

$$
\dot{y}(t)=L y(t)=\frac{1}{n} A y(t)
$$

Where the form of $L=\frac{1}{n} A$ is going to ensure that the equation is independent from the number of participants.

To interpret Jacobian $\frac{1}{n} A$ we assume that $a_{i j}$ is the strength of action by which $j^{\text {th }}$ participant affects the $i^{\text {th }}$ participant. Then $i^{\text {th }}$ participant is influenced by average effect of all of the participants.

Denote $P_{k}=\left(p_{i j}\right)$ the projector matrix whose entries are zero except $p_{k k}=1$ and similarly let $A_{i j}$ be the matrix whose entries are zero except the entry $a_{i j}$. Then

$$
A=\sum_{i=1}^{n} \sum_{j=1}^{n} A_{i j}=\sum_{i=1}^{n} \sum_{j=1}^{n} P_{i} A P_{j}=\left(\sum_{i=1}^{n} P_{i}\right) A\left(\sum_{j=1}^{n} P_{j}\right)
$$

where $A_{i j}=P_{\mathrm{i}} A P_{\mathrm{j}}$. 

can be written as

The elementary equation of index $(i, j) \dot{y}_{i}=a_{i j} y_{j}$

$$
P_{i} \dot{y}=P_{i} A P_{j} y
$$

Thus for the sum all of $n^{2}$ elementary equations

$$
n\left(\sum_{i=1}^{n} P_{i}\right) \dot{y}=\left(\sum_{i=1}^{n} P_{i}\right) A\left(\sum_{j=1}^{n} P_{j}\right) y
$$

that is

$$
\dot{y}=\frac{1}{n} A y
$$

Which means that our main equation can be stated as sum of $n^{2}$ elementary equations.

\section{b) Role of randomness}

To have a rough picture, we assume that the participants form some groups consisting of similarly behaving elements. Consequently the system matrix $A$ consists of some blocks whose entries are independent random variables having the same expected values. We collected these expected values into the density matrix. Thus block random system has two important parameters: the density matrix and the dispersion of the entries. In this case the system matrix is called block random matrix.

It is proved that spectra of block random matrices consists of a fixed number of $\mathrm{n}^{\text {th }}$ order eigenvalues while most of the eigenvalues are of order $\sqrt{ } n$ around zero. From the point of view of our investigations, the most important case is slight unstable block random matrix which means that the large in absolute value eigenvalues of the block random matrix have negative real parts.

\section{ili. Effects of the Density Matrix and Dispersion}

To characterize behavior of block random systems we divided the envelope of the bundle of trajectories into two parts, that is, into a convergence period and a divergence period of time [2]

If lengths of these periods, that is, convergence time and divergence time are suitable long then the participants are able to adapt themselves to conditions. In this case we are faced to a so called structural cycle and it is possible to start another cycle of the original nonlinear system.

If either convergence period or divergence period or both are too short, a certain part of the participants are unable to adapt themselves to circumstances and so crisis appears.

\section{Consequences in Economics}

Statistical data show that most of enterprises are charged with repayable loan. Consequently, if base rate is raised then strengths of interactions between participants decrease. This can be taken into consideration by proportional reduction (multiplication by a positive number less than 1) of the density matrix. This results in a shorter cycle time, that is, in quicker changes which in some circumstances can turn into crises.

Furthermore, it is reasonable to assume that a higher base rate causes increased differences between participants, that is, results in a greater dispersion. Thus base rate influences both parameters - density matrix and dispersion - in one direction from the viewpoint of speed of changes [2 Table 1].

The appearance of crisis can be avoided if base rate is kept in an appropriate low level. In this case, repetitive so called structural cycles can be observed and the system is free from crises [2 Figure 9].

In general, one has to think about aims of economic policies. If we leave the paradigm of growth and instead we follow the paradigm of crisis avoidance then development is realized in a more natural way with less troubles.

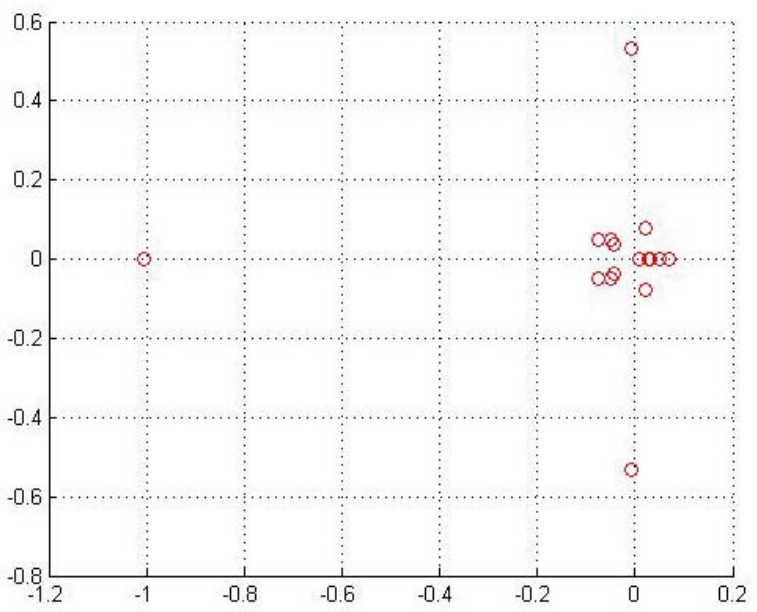

Figure 1: 16 Eigenvalues of a slightly unstable block random system

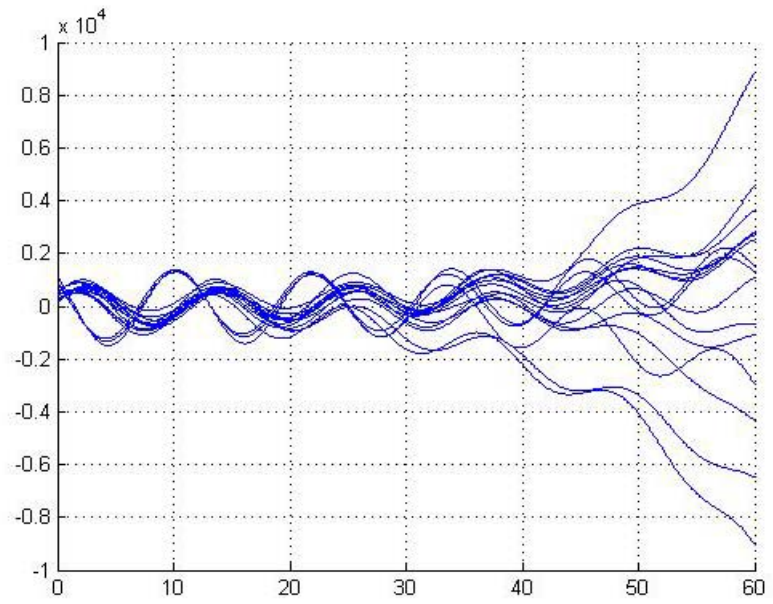

Figure 2: Trajectories of profits of 16 participants 


\section{SUMMARY}

Block random matrix has two important parameters, expected values of the entries which are collected into the density matrix and dispersion of the entries which is assumed to be a certain value. The connection between base rate and parameters of block random systems require further investigations. However, we emphasize that a higher base rate entails decreasing the magnitude of the density matrix as well and a larger standard deviation of the entries. Thus the alteration of both parameters results in faster processes which allow the crisis to appear. Otherwise an appropriate low level of interests makes it possible the participants to adapt themselves to the fundamentally nonlinear system and thus to avoid crisis.

It seems clear that block random model is able to explain why economy is doing well with a relatively low level of base rate and crashes often in case of systems with high level of interests.

\section{References Références Referencias}

1. Juhász, F. (2016) Crisis cycles in slightly unstable block random model, Journal of Statistical Computation and Simulation, vol. 86, pp. 3270-3286

2. Juhász, F. (2020) Structural cycles in slightly unstable block random model, Journal of Statistics \& Management Systems, DOI: 10.1080/09720510. 2020.1714872 